\title{
EVN and MERLIN observations of nearby BL Lac objects and multiwavelength analysis
}

Marcello Giroletti*1 and Gabriele Giovannini ${ }^{1,2}$

${ }^{1}$ INAF Istituto di Radioastronomia, via Gobetti 101, 40129 Bologna, Italy

${ }^{2}$ University of Bologna, Department of Astronomy, via Ranzani 1, 40127 Blogna, Italy

E-mail: giroletti@ira.inaf.it, ggiovanneira.inaf.it

We present new observations of BL Lacertae objects with the EVN and/or MERLIN, thus exploring their radio morphologies from sub-parsec to kiloparsec scales. We further consider broadband data from the Hubble Space Telescope and X-ray satellites, discussing the radiation mechanisms, the black hole mass, and the fundamental plane of the $\mathrm{BH}$.

8th European VLBI Network Symposium

September 26-29, 2006

Toruń, Poland

${ }^{*}$ Speaker. 

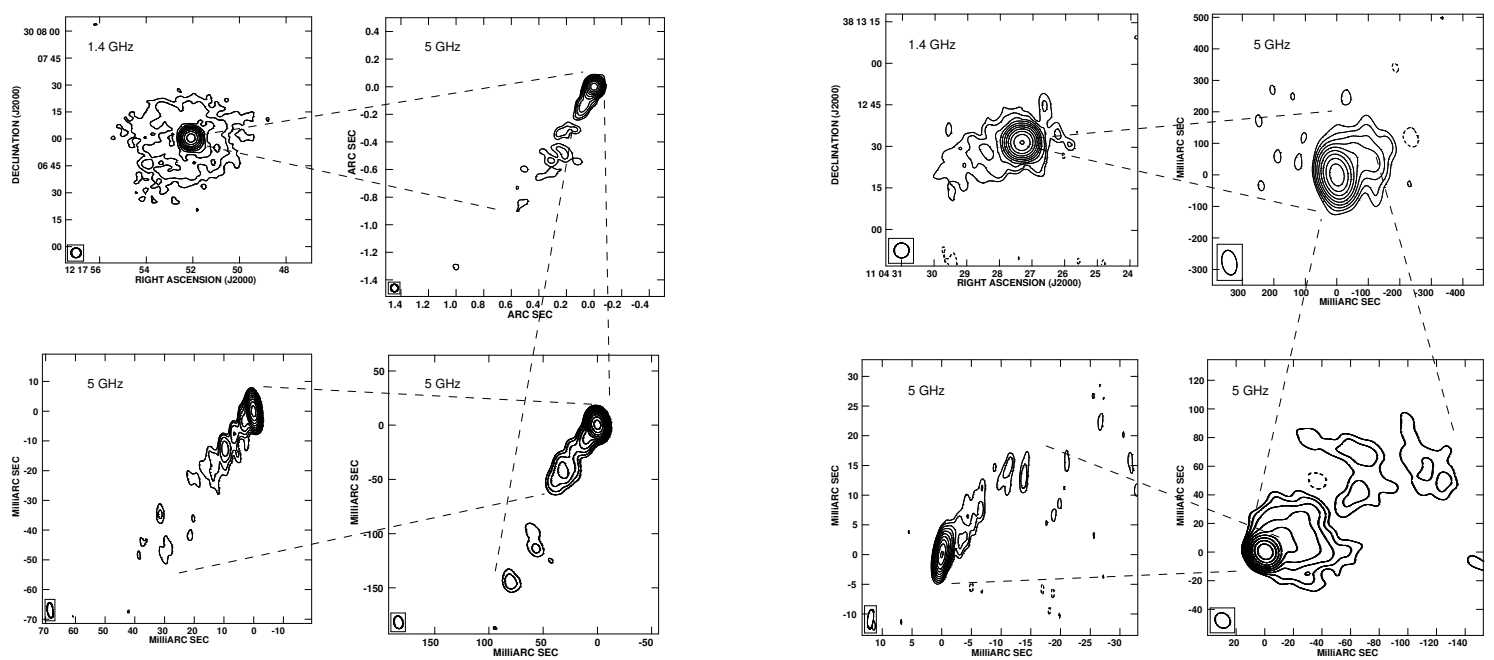

Figure 1: Clockwise from top left: VLA, MERLIN, EVN+MERLIN and EVN only images for $1215+303$ (left) and Mrk 421 (right)

BL Lac objects are Active Galactic Nuclei whose basic radio properties are as follows: flat spectrum, compactness, variability, core dominance, and strong polarization. According to unified schemes, they are expected to be the beamed counterparts of FR I radio galaxies. Because of relativisting boosting, the parsec-scale properties of BL Lacs and FR Is are apparently different. In order to derive Doppler factors for BL Lacs and to provide support for unified schemes, we selected a sample of 29 nearby $(z<0.2)$ objects with HST images and we observed it with radio interferometers at arcsecond and milliarcsecond resolution $[1,2]$.

\section{Radio observations}

Nine BL Lac objects were observed at high resolution with the EVN. Their parsec-scale images reveal an almost ubiquitous presence of one-sided jets. Under the assumption of intrinsically symmetric jets, this provides evidence for relativistic velocities in the parsec-scale region of jets. This result supports the previous findings for the same sample [1], and we derive Lorentz factors in the sample clustered around $\Gamma=4$. In the three sources observed also with MERLIN, i.e. with additional short baselines, we trace the jet out to several hundred parsecs, and still find no hint of counter-jets. On kiloparsec scales, the structure of 1728+502 remains aligned with the inner region direction, while $1215+303$ and Mrk 421 reveal a symmetric structure without a clear connection to the parsec-scale jet (see Fig. 1).

In Mrk 421, we have also evidence of limb brightening of the jet, from the very inner regions out to $\sim 30$ mas, similarly to the other TeV source in the sample (i.e. Mrk 501 [3]). This result is of great importance for the development of jet launch models and the study of the jet-environment interaction.

\section{Multiwavelength analysis}

Thanks to the availability of X-ray fluxes and high resolution optical (HST) data for the whole 

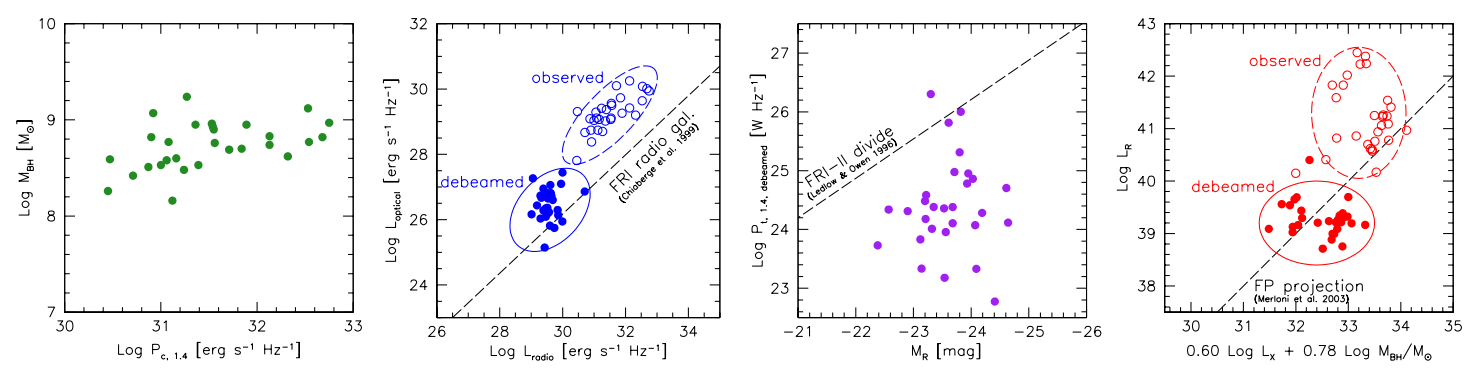

Figure 2: Multiwavelength data plots. See text for discussion.

sample, we carried out a multiwavelength analysis. In particular, by resolving the host galaxy and separating the non-thermal core, we can understand some physical properties of the BL Lac phenomenon. Given the distance-limited nature of the present sample, we are confident of the robustness of our results, which we illustrate in Fig. 2 and summarize as follows:

1. There is a significant correlation between the mass of the central SMBH and the radio luminosity, both nuclear and extended, regardless of any common distance dependence. However, there is a large scatter in the correlation, so that radio luminosity alone can not be used as a measure of the $\mathrm{BH}$ mass.

2. Radio and optical nuclear luminosities (and fluxes) show a strong correlation, indicating a common origin for the emission at both wavelengths, i.e. non-thermal synchrotron radiation. Moreover, if we de-beam the observed luminosities, the luminosities of the cores are ruled by the same correlation as that found for low power radio galaxies [4], in strong support of the unification of BL Lacs and FR Is based on the orientation.

3. Still in support of the BL Lac/FR I unification, our sources lie below the dividing line in the Ledlow \& Owen diagram [5]; for any given $M_{\mathrm{BH}}$ (estimated from the host galaxy magnitude), low redshift BL Lacs have lower radio power than FR II radio galaxies with similar $M_{\mathrm{BH}}$.

4. The radio and X-ray luminosities do not follow the fundamental plane of BH activity found for other super-massive and galactic BH [6], clearly because of the relevant Doppler boosting of the jet. However, when we consider de-beamed quantities, the FP relation becomes quite well-reproduced, although some overall scatter and the LBL-HBL offset appear.

\section{References}

[1] Giroletti M. et al. 2004b, ApJ 613, 752

[2] Giroletti M. et al. 2006, ApJ 646, 801

[3] Giroletti M. et al. 2004a, ApJ 600, 127

[4] Chiaberge, M., Capetti, A., \& Celotti, A. 1999, A\&A, 349, 77

[5] Ledlow, M. J., \& Owen, F. N. 1996, AJ, 112, 9

[6] Merloni, A., Heinz, S., \& di Matteo, T. 2003, MNRAS, 345, 1057 